\title{
VARIABILIDAD GENÉTICA DE TRES ESPECIES CUBANAS DEL GÉNERO Peltophryne (ANURA: BUFONIDAE)
}

\section{GENETIC VARIABILITY OF THREE CUBAN SPECIES OF THE GENUS Peltophryne (ANURA: BUFONIDAE)}

\author{
RIVALTA-G., VILMA ${ }^{1^{*}}$ M.Sc., BEROVIDES-Á., VICENTE ${ }^{2}$.Dr.C., RODRÍGUEZ- \\ S., LOURDES ${ }^{1}$ Dr.C., CHAMIZO-L., ADA ${ }^{1}$. M.Sc. \\ ${ }^{1}$ Instituto de Ecología y Sistemática, CITMA, C. de Varona Km 3.5, Boyeros. CP \\ 10800, AP 8029, La Habana, Cuba. ${ }^{2}$ Facultad de Biología, Universidad de La \\ Habana. 25 Y J Vedado, CP 10400, La Habana, Cuba. \\ *Correpondencia: zoologia.ies@ama.cu
}

\section{Resumen}

El género Peltophryne está representado en Cuba por ocho especies, todas endémicas. En este trabajo se analizan 10 poblaciones de $P$. peltocephala, dos de $P$. fustiger y una de $P$. florentinoi. Para el análisis de las relaciones genéticas, se calcularon las distancias genéticas entre poblaciones y especies según el índice de Nei (1972). Se realizó la electroforesis en gel de almidón para cinco enzimas y las proteínas totales del músculo, las cuales están codificadas para 12 loci. Seis loci resultaron monomórficos y fijados para el mismo alelo en todas las poblaciones y especies, cuatro polimórficos y dos con carácter diagnóstico. Los cuatro sistemas polimórficos se encuentran en equilibrio de Hardy-Weinberg en cada población y área geográfica. En la Isla de la Juventud se encuentran los mayores valores de heterocigosidad (Hobs $=0.117$ y 0.077 ) para $P$. peltocephala. El dendrograma derivado de la variación enzimática y proteínica mostró que $P$. fustiger y $P$. peltocephala difieren con un valor promedio de $\mathrm{D}=0.098$, mientras $P$. florentinoi difiere de ambos en una $D=0.197$. $P$. florentinoi presentó un alelo fijado (LDH-B) y un alelo único (PT-B). Las poblaciones de $P$. peltocephala de la Isla de la Juventud se separan del resto, en un valor promedio de $D=0.019$ y estos resultados sugieren que pudieran ser tratadas como subespecies.

Palabras claves: Bufo, sapos, sistemática bioquímica, isoenzimas, Cuba. 


\section{Abstract}

The genus Peltophryne is represented in Cuba with eight endemic species. In this paper, 10 populations of $P$. peltocephala, two of $P$. fustiger, and one of $P$. florentinoi are analyzed. For the genetic relations analysis, the genetic distances among populations and species were calculated by using Nei's index (1972). Electrophoresis in starch gel was made for five enzymes and total muscle proteins which are codified for 12 loci. Six of them were monomorphic and fixed for the same allele in all of the species and populations, four were polymorphic and two with a diagnostic character. The four polymorphic systems were in HardyWeinberg's equilibrium in every population and geographic area. The highest values of heterozigosity (Hobs $=0.117$ y 0.077 ) for $P$. peltocephala were obtained from the Isla de la Juventud (youth island). The cluster obtained from the enzymatic and protein variability showed that $P$. peltocephala and $P$. fustiger differed with an average value of $D=0.098$ while $P$. florentinoi differed from the two above with $\mathrm{D}=0.197$. $P$. florentinoi showed a fixed allele (LDH-B) and an unique allele (PT-B). The populations of $P$. peltocephala from the Isla de la Juventud (youth island) differed from the rest with an average value of $D=0.019$, what suggests that they could be recognized as subspecies.

Key words: Peltophryne, P. florentinoi, P. fustiger, P. peltocephala

\section{Introducción}

Los anfibios cubanos se componen actualmente de 62 especies de ranas y sapos (DÍAZ y CÁDIZ, 2008). Estas especies pertenecen al orden Anura y se encuentran agrupadas en las familias Bufonidae, Leptodactylidae, Hylidae y Ranidae, con un género cada una de ellas y $95,2 \%$ de endemismo.

PREGILL (1981) resucitó el género Peltophryne Fitzinger, 1843, para los sapos antillanos, sobre la base de los huesos craneales. Sin embargo, HEDGES (1996), teniendo en cuenta las distancias inmunológicas en albúmina, PRAMUK (2000) y PRAMUK et al. (2001), a partir del análisis de los huesos prenasales, de las relaciones evolutivas y de la biogeografía, consideraron que la separación de Bufo y Peltophryne no se justificaba para dichas especies. No obstante, FROST et al. (2006), PRAMUK (2006) y PRAMUK et al. (2007) retomaron el género Peltophryne sobre la investigación de mayor número de especies y de caracteres que en los trabajos anteriores. Por tanto, el criterio taxonómico más actual y riguroso es el del género Peltophryne hasta tanto no se demuestre lo contrario. 
Los sapos de Las Antillas Mayores están constituidos por 12 especies distribuidas en tres de ellas. Ocho están presentes en Cuba: Peltophryne cataulaciceps (Schwartz, 1959), P. empusa Cope, 1862, P. gundlachi (Ruibal, 1959), P. longinasa (Stejneger, 1905), P. peltocephala (Tschudi, 1838), P. fustiger (Schwartz, 1960), P. taladai (Schwartz, 1960) y P. florentinoi (Moreno y Rivalta, 2007), todas endémicas de Cuba. Tres especies son de La Española: $P$. fluviatica (Schwartz, 1972), P. guentheri (Cochran, 1941) y P. fracta (Schwartz, 1972). Una sola es de Puerto Rico: P. lemur Cope 1868 (1869) (POWELL et al., 1996).

La separación electroforética de las enzimas y proteínas no enzimáticas es una técnica útil y complementaria para los estudios taxonómicos (AVISE, 1994; HEDRICK, 2000; LOWE et al. 2004). Dado que no existen publicaciones sobre el uso de esta técnica para los bufónidos cubanos, nos propusimos caracterizar desde el punto de vista genético, tres especies de sapos $(P$. peltocephala, $P$. fustiger y $P$. florentinoi), por medio del polimorfismo de cinco sistemas enzimáticos y las proteínas totales del músculo, determinando el equilibrio genético, el grado de polimorfismo, la variabilidad genética, el flujo genético y las distancias genéticas.

\section{Materiales y métodos}

Se analizaron mediante electroforesis 186 ejemplares procedentes de diversas localidades del país, capturados a mano entre 1988 y 1992, pertenecientes a 10 poblaciones de $P$. peltocephala, dos de $P$. fustiger y una de $P$. florentinoi (Fig. 1). Los ejemplares se trasladaron al laboratorio en bolsas de tela humedecida y se anestesiaron con éter antes de proceder a la disección. Se les realizó la extracción de diversos tejidos: músculo, hígado y corazón y se homogeneizaron con agua en igual proporción peso-volumen $(\mathrm{P} / \mathrm{V})$. Los extractos de tejidos se analizaron por el método de electroforesis horizontal en gel de almidón, según la técnica descrita por SMITHIES (1955).

Se emplearon tres sistemas de buffer para la preparación electroforética de cinco enzimas y las proteínas totales, buffer A con pH 6.1 (CLAYTON y TRETIAK, 1972), buffer B con pH 6.7 (SELANDER et al, 1971) y buffer $C$ con pH 8.1 (RIDGWAY et al., 1970). Las enzimas y proteínas no enzimáticas utilizadas fueron: Aspartato aminotransferasa (AAT-A y AAT-B), Isocitrato deshidrogenasa (IDH-A e IDH-B), Lactato deshidrogenasa (LDH-A y LDH-B), Malato 
deshidrogenasa (MDH-A y MDH-B), Fosfoglucomutasa (PGM) y Proteínas totales (PT-A, PT-B y PT-C).

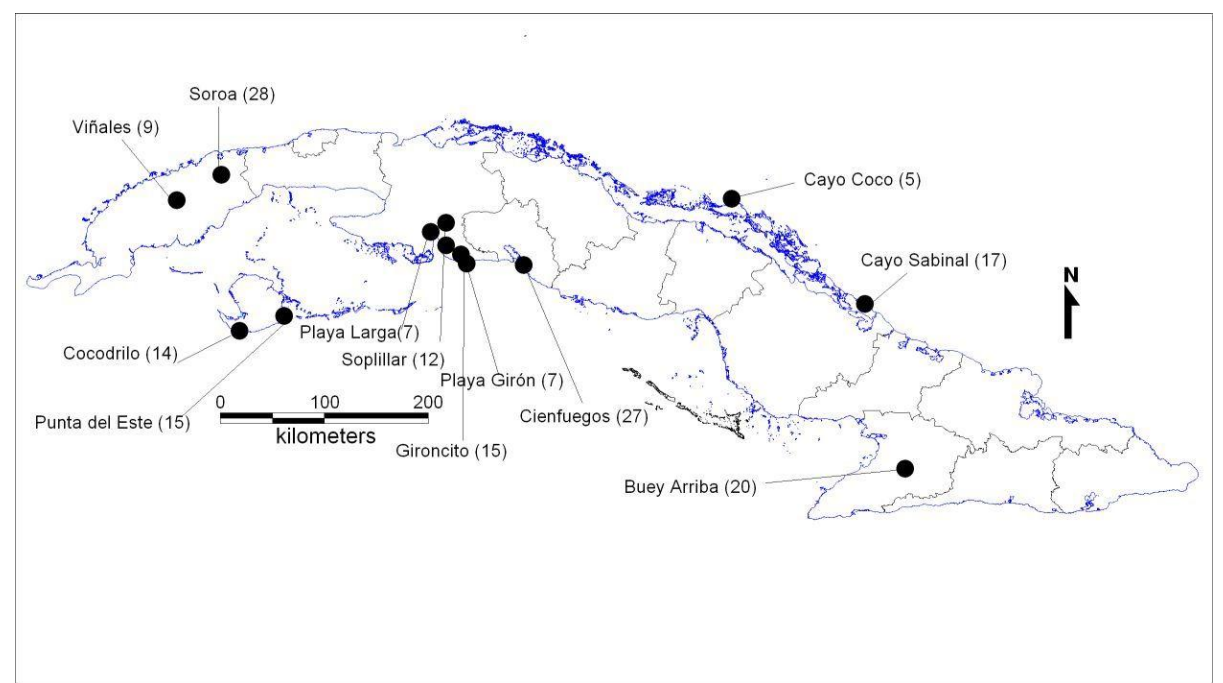

Figura 1. Área de trabajo.

La mayoría de las coloraciones, con excepción de la aspartato aminotransferasa y las proteínas totales del músculo, se realizaron en agar (BREWER, 1970). La tinción de las enzimas siguió el método de SHAW y PRASAD (1970) y HARRIS y HOPKINSON (1976). La detección de las proteínas totales del músculo se realizó utilizando azul de Coomasie $\mathrm{G}$ al $1 \%$ en ácido tricloroacético al $12 \%$.

En el análisis estadístico, los niveles de significación utilizados para definir las probabilidades de rechazo de las hipótesis nulas utilizadas fueron las convencionales: $\left.\mathrm{P}<0.05\left(^{*}\right), \mathrm{p}<0.01{ }^{* *}\right)$ y $\left.\mathrm{P}<0.001{ }^{* * *}\right)$

Los genotipos individuales se usaron para calcular las frecuencias alélicas en cada población y se determinó el equilibrio de Hardy-Weinberg (HEDRICK, 2000) por la prueba de $\mathcal{X}^{2}$. Las frecuencias génicas se compararon mediante una prueba $\mathrm{G}$ según SOKAL y ROLF (1981).

Se establecieron cinco áreas geográficas para $P$. peltocephala debido a que el tamaño de muestra por población es pequeño: Cayos del Archipiélago SabanaCamagüey (Cayo Sabinal y Cayo Coco), Isla de la Juventud (Punta del Este y Cocodrilo), Ciénaga de Zapata (Playa Larga, Playa Girón, Soplillar y Los Sábalos), Cienfuegos y Buey Arriba. Para estas áreas se compararon sus frecuencias 
génicas mediante la prueba G. También se compararon las frecuencias génicas entre especies.

El grupo de metapoblaciones está formado por: la Isla de la Juventud (Punta del Este y Cocodrilo), los Cayos (Cayo Sabinal y Cayo Coco) y la Ciénaga de Zapata (Playa Larga, Playa Girón Soplillar y Los Sábalos).

Se determinaron las siguientes variables genéticas:

P: grado de polimorfismo (\% de loci polimórficos) = Número de loci polimórficos/Número de loci examinados.

A: Diversidad alélica (alelos por locus) $=\Sigma$ alelos de todos los loci en cada población/Número total de loci examinados en esa población.

Ho: Heterocigosidad observada $=$ Número de fenotipos heterocigóticos/Número total de fenotipos.

Se determinaron los índices de diversidad genética siguientes: $\mathrm{Ht}=$ heterocigosidad total, $\mathrm{Hp}=$ heterocigosidad media poblacional, Dst = diferencia entre las heterocigosidades de las poblaciones, Gst = diversidad genética relativa y Nem = flujo genético. Estos cálculos se realizaron según NEI (1987).

$\mathrm{Ht}$ se define matemáticamente como: $\mathrm{Ht}=\mathrm{Hp}+$ Dst.

Gst como: Gst $=$ Dst $/ \mathrm{Ht}$.

El coeficiente de diferenciación genética, Gst, da una medida de la contribución que hace la diversidad genética entre poblaciones, (Dst) a la diversidad genética total de la especie $(\mathrm{Ht})$. Este valor varía de cero a uno y es altamente dependiente del valor $\mathrm{Ht}$. Cuando éste es pequeño, Gst puede ser grande incluso si la diferenciación genética absoluta es pequeña.

El flujo genético (Nem) es la cantidad de individuos que migran por generación y se define como: Nem $=(1 /$ Gst- 1$) / 4$.

Sí Nem es mayor que 1 se supone que las poblaciones mantienen un cierto intercambio genético. Si es menor que 0 estas poblaciones no están interconectadas y deben manejarse como poblaciones independientes. 
Para el análisis de la estructura genética se utilizó el modelo isla (WRIGHT, 1965), teniendo en cuenta que casi todas las metapoblaciones estudiadas estuvieron integradas por sólo dos subpoblaciones. Se asume entonces que entre estas subpoblaciones existe la misma probabilidad de intercambiar individuos equitativamente, lo cual precisamente constituye una de las premisas para la aplicación del modelo isla.

Para el análisis de las relaciones genéticas, se calcularon las distancias genéticas entre poblaciones y especies según el índice de NEI (1972). A partir de estas distancias genéticas se construyó una matriz, la cual sirvió de base para realizar un análisis de agrupamiento siguiendo el algoritmo UPGMA (SNEATH y SOKAL, 1973). Estos resultados se presentaron de forma gráfica en un dendrograma, donde se representan las relaciones genéticas estimadas entre estos taxones, utilizando para ello el programa NTSYS (1993).

\section{Resultados}

Frecuencias génicas. Como 6 de los 12 loci presentan un solo alelo, las frecuencias génicas se describen para los restantes loci (PGM, AAT-B, LDH-B, MDH-A, PT-B y PT-C) (Tabla 1). En general, de dos a cinco variantes alélicas se presentan en cada uno de los loci polimórficos.

Comparación de las frecuencias génicas. Entre las cinco áreas geográficas de P. peltocephala (Tabla 2) el locus PGM diferencia a las poblaciones de la Isla de la Juventud (Punta del Este y Cocodrilo) del resto y en el locus AAT-B hay diferencias génicas entre ellas. La mayoría de las poblaciones presentan el alelo $\underline{b}$ en baja frecuencia o carecen de él para el locus LDH-B, con diferencias entre las áreas, por lo que se establecen tres grupos: 1- Cayos y Buey Arriba; 2Cienfuegos; y 3-Ciénaga de Zapata e Isla de la Juventud. No hay diferencias para el locus MDH-A entre áreas.

Entre las especies, el locus PGM diferencia a $P$. florentinoi de $P$. peltocephala y $P$. fustiger. (Tabla 3) Para el locus AAT-B también hay diferencias significativas entre las especies: $P$. florentinoi no presenta el alelo $\underline{c}$. El locus $L D H-B$ diferencia a $P$. florentinoi de $P$. peltocephala y $P$. fustiger por tener fijado el alelo $\underline{b}$. El locus MDHB diferencia a $P$. fustiger de $P$. peltocephala y $P$. florentinoi por tener el alelo a con mayor frecuencia. 
Tabla 1. Frecuencias génicas en 13 poblaciones de sapos. Localidades: Soroa (SR); Viñales (VI); Sabinal (SA); Cayo Coco (CC); Punta del Este (PE); Cocodrilo (CO); Playa Girón (PG); Playa Larga (PL); Soplillar (SP); Los Sábalos (LS); Cienfuegos (CF); Buey Arriba (BA); Gironcito (GI). Entre paréntesis, tamaño de la muestra.

\begin{tabular}{|c|c|c|c|c|c|c|c|c|c|c|c|c|c|c|}
\hline \multirow[b]{2}{*}{ Locus } & \multirow[b]{2}{*}{ Alelos } & \multicolumn{2}{|c|}{ P. fustiger } & \multicolumn{10}{|c|}{ P. peltocephala } & \multirow{2}{*}{$\begin{array}{c}P . \\
\text { florentino } \\
\text { Gl } \\
(15)\end{array}$} \\
\hline & & $\begin{array}{l}\text { SR } \\
(28)\end{array}$ & $\begin{array}{l}\mathrm{VI} \\
\text { (9) }\end{array}$ & $\begin{array}{l}\text { SA } \\
\text { (17) }\end{array}$ & $\begin{array}{l}\mathrm{CC} \\
(5)\end{array}$ & $\begin{array}{l}\mathrm{PE} \\
\text { (15) }\end{array}$ & $\begin{array}{l}\mathrm{CO} \\
(14)\end{array}$ & $\begin{array}{l}\mathrm{PG} \\
(7)\end{array}$ & $\begin{array}{l}\mathrm{PL} \\
\text { (7) }\end{array}$ & $\begin{array}{l}S P \\
(12)\end{array}$ & $\begin{array}{l}\text { LS } \\
\text { (10) }\end{array}$ & $\begin{array}{l}\mathrm{CF} \\
(27)\end{array}$ & $\begin{array}{l}\text { BA } \\
(20)\end{array}$ & \\
\hline \multirow[t]{5}{*}{ PGM } & $a$ & 0.000 & 0.000 & 0.000 & 0.000 & 0.000 & 0.000 & 0.000 & 0.000 & 0.000 & 0.050 & 0.000 & 0.000 & 0.000 \\
\hline & b & 0.036 & 0.000 & 0.000 & 0.100 & 0.000 & 0.000 & 0.000 & 0.000 & 0.000 & 0.000 & 0.026 & 0.075 & 0.000 \\
\hline & C & 0.875 & 0.944 & 1.000 & 0.900 & 0.467 & 0.607 & 1.000 & 1.000 & 0.875 & 0.900 & 0.974 & 0.875 & 1.000 \\
\hline & $d$ & 0.071 & 0.056 & 0.000 & 0.000 & 0.367 & 0.036 & 0.000 & 0.000 & 0.000 & 0.000 & 0.000 & 0.050 & 0.000 \\
\hline & $e$ & 0.018 & 0.000 & 0.000 & 0.000 & 0.166 & 0.357 & 0.000 & 0.000 & 0.125 & 0.050 & 0.000 & 0.000 & 0.000 \\
\hline \multirow[t]{3}{*}{ AAT-B } & $a$ & 0.071 & 0.000 & 0.147 & 0.000 & 0.000 & 0.000 & 0.000 & 0.000 & 0.000 & 0.000 & 0.000 & 0.050 & 0.200 \\
\hline & $\mathrm{b}$ & 0.840 & 0.944 & 0.853 & 1.000 & 1.000 & 1.000 & 1.000 & 1.000 & 1.000 & 1.000 & 0.952 & 0.900 & 0.800 \\
\hline & C & 0.089 & 0.056 & 0.000 & 0.000 & 0.000 & 0.000 & 0.000 & 0.000 & 0.000 & 0.000 & 0.048 & 0.050 & 0.000 \\
\hline \multirow[t]{2}{*}{ LDH-B } & $a$ & 0.911 & 0.889 & 1.000 & 1.000 & 0.700 & 0.714 & 1.000 & 0.857 & 0.792 & 0.900 & 0.925 & 1.000 & 0.000 \\
\hline & $\mathrm{b}$ & 0.089 & 0.111 & 0.000 & 0.000 & 0.300 & 0.286 & 0.000 & 0.143 & 0.208 & 0.100 & 0.075 & 0.000 & 1.000 \\
\hline \multirow[t]{2}{*}{ MDH-A } & $a$ & 0.893 & 1.000 & 1.000 & 1.000 & 1.000 & 1.000 & 1.000 & 1.000 & 1.000 & 1.000 & 0.978 & 0.975 & 0.967 \\
\hline & $\mathrm{b}$ & 0.107 & 0.000 & 0.000 & 0.000 & 0.000 & 0.000 & 0.000 & 0.000 & 0.000 & 0.000 & 0.022 & 0.025 & 0.033 \\
\hline \multirow[t]{2}{*}{ PT-B } & $a$ & 1.000 & 1.000 & 1.000 & 1.000 & 1.000 & 1.000 & 1.000 & 1.000 & 1.000 & 1.000 & 1.000 & 1.000 & 0.000 \\
\hline & $\mathrm{b}$ & 0.000 & 0.000 & 0.000 & 0.000 & 0.000 & 0.000 & 0.000 & 0.000 & 0.000 & 0.000 & 0.000 & 0.000 & 1.000 \\
\hline \multirow[t]{2}{*}{ PT-C } & $a$ & 1.000 & 1.000 & 0.000 & 0.000 & 0.000 & 0.000 & 0.000 & 0.000 & 0.000 & 0.000 & 0.000 & 0.000 & 0.000 \\
\hline & $\mathrm{b}$ & 0.000 & 0.000 & 1.000 & 1.000 & 1.000 & 1.000 & 1.000 & 1.000 & 1.000 & 1.000 & 1.000 & 1.000 & 1.000 \\
\hline
\end{tabular}

Tabla 2. Comparación de las frecuencias génicas por áreas geográficas en $P$. peltocephala. G, valor de G calculado; ***, significativo para $\mathrm{p}<0.001$; ${ }^{* *}$, significativo para $p<0.01 ;$ n.s., no significativo.

\begin{tabular}{|c|c|c|c|c|c|c|c|}
\hline Locus & Alelos & Cayos & $\begin{array}{c}\text { I. de la } \\
\text { Juventud }\end{array}$ & $\begin{array}{c}\text { C. de } \\
\text { Zapata }\end{array}$ & Cienfuegos & Buey Arriba & G \\
\hline PGM & $\mathrm{a}$ & 0.00 & 0.00 & 0.01 & 0.00 & 0.00 & \\
\hline & $\mathrm{b}$ & 0.02 & 0.00 & 0.00 & 0.03 & 0.08 & \\
\hline & $\mathrm{c}$ & 0.98 & 0.53 & 0.93 & 0.97 & 0.87 & \\
\hline & $\mathrm{d}$ & 0.00 & 0.21 & 0.00 & 0.00 & 0.05 & \\
\hline & $\mathrm{e}$ & 0.00 & 0.26 & 0.06 & 0.00 & 0.00 & $51.78^{\star * *}$ \\
\hline AAT-B & $\mathrm{a}$ & 0.11 & 0.00 & 0.00 & 0.00 & 0.05 & \\
\hline & $\mathrm{b}$ & 0.89 & 1.00 & 1.00 & 0.95 & 0.90 & \\
\hline & $\mathrm{c}$ & 0.00 & 0.00 & 0.00 & 0.05 & 0.05 & $17.52^{* \star}$ \\
\hline LDH-B & $\mathrm{a}$ & 1.00 & 0.73 & 0.87 & 0.92 & 1.00 & \\
\hline & $\mathrm{b}$ & 0.00 & 0.27 & 0.13 & 0.08 & 0.00 & $30.96^{\star \star *}$ \\
\hline MDH-A & $\mathrm{a}$ & 1.00 & 1.00 & 1.00 & 0.98 & 0.98 & \\
\hline & $\mathrm{b}$ & 0.00 & 0.00 & 0.00 & 0.02 & 0.02 & 4.90 n.s. \\
\hline
\end{tabular}


Tabla 3. Comparación de las frecuencias génicas por especies. G, valor de G calculado; ${ }^{* *}$, significativo para $p<0.001 ;{ }^{* *}$, significativo para $p<0.01 ;$, significativo para $p<0.05$; n.s., no significativo.

\begin{tabular}{|c|c|c|c|c|c|}
\hline Locus & Alelos & P. peltocephala & P. fustiger & P. florentinoi & G \\
\hline PGM & a & 0.00 & 0.00 & 0.00 & \\
\hline & b & 0.02 & 0.03 & 0.00 & \\
\hline & c & 0.84 & 0.89 & 1.00 & \\
\hline & d & 0.06 & 0.07 & 0.00 & \\
\hline & e & 0.08 & 0.01 & 0.00 & $14.02^{*}$ \\
\hline AAT-B & a & 0.03 & 0.05 & 0.20 & \\
\hline & b & 0.96 & 0.87 & 0.80 & \\
\hline & c & 0.01 & 0.08 & 0.00 & $20.11^{\text {*** }}$ \\
\hline LDH-B & a & 0.87 & 0.91 & 0.00 & \\
\hline & b & 0.13 & 0.09 & 1.00 & $117.45^{\text {*** }}$ \\
\hline MDH-B & a & 0.99 & 0.92 & 0.97 & \\
\hline & b & 0.01 & 0.08 & 0.03 & $10.43^{\text {** }}$ \\
\hline
\end{tabular}

Equilibrio genético. Se comprobó en cada locus polimórfico el equilibrio de Hardy-Weinberg y no se encontró divergencia significativa entre los valores esperados y los observados, tanto para las 13 poblaciones como para las cinco áreas geográficas de $P$. peltocephala.

Grado de polimorfismo. En relación con P. peltocephala cuando se calcula este parámetro se observa una gran variabilidad, lo cual es común para este índice (Tabla 4). Por esta razón el mejor estimado de $\mathrm{P}$ es por áreas geográficas, en las cuales los valores varían entre 16.6 y $33.3 \%$. Soroa y Cienfuegos presentaron una mayor proporción de loci polimórficos, en comparación con las otras poblaciones, indicando una mayor variación genética de $P$. fustiger y $P$. peltocephala, respectivamente. $P$. florentinoi registra un valor de $\mathrm{P}$ menor que las otras dos especies.

Heterocigocidad. En $P$. peltocephala la Hobs varía entre 0 y 0.117 ; en $P$. fustiger, entre 0.037 y 0.074 y en $P$. florentinoi es de 0.039 . La heterocigosis promedio es menor en $P$. peltocephala que en $P$. fustiger (Tabla 5). En cuanto a las áreas seleccionadas para $P$. peltocephala, la Isla de la Juventud es la de mayor Hobs, mientras que la de los Cayos tiene el valor menor.

Descomposición de la heterocigosidad por metapoblación en $\boldsymbol{P}$. peltocephala. La mayor heterocigosidad total $(\mathrm{Ht})$ la tiene Isla de la Juventud (8.4\%), le sigue Ciénaga de Zapata $(2.5 \%)$ y la menor es la de los Cayos con $1.9 \%$. La heterocigosidad media poblacional $(\mathrm{Hp})$ sigue el mismo patrón que la anterior: el valor mayor le corresponde a la Isla de la Juventud con $8.1 \%$, el menor a los cayos con $1.8 \%$, y la Ciénaga de Zapata con $2.4 \%$. La diferencia entre las 
heterocigosidades de las poblaciones (Dst) varió entre 0.001 (Cayos y Ciénaga de Zapata) y 0.003 (Isla de la Juventud).

Tabla 4. Grado de polimorfismo $(\mathrm{P})$ por población, área geográfica y especie del género Peltophryne. N, tamaño de la muestra.

\begin{tabular}{|c|c|c|c|c|c|}
\hline & & & \multicolumn{3}{|c|}{$\mathbf{P i}(\%)$} \\
\hline Población & $\mathbf{N}$ & $\begin{array}{l}\text { No. de Loci } \\
\text { Polimórficos }\end{array}$ & Población & Área geográfica & Especie \\
\hline Soroa & 28 & 4 & 33.3 & - & P. fustiger \\
\hline Viñales & 9 & 3 & 25.0 & - & 29.1 \\
\hline Cayo Sabinal & 17 & 1 & 8.3 & - & P. peltocephala \\
\hline Cayo Coco & 5 & 1 & 8.3 & 16.6 & 25.0 \\
\hline Punta del Este & 15 & 2 & 16.6 & - & \\
\hline Cocodrilo & 14 & 2 & 16.6 & 16.6 & \\
\hline Playa Girón & 7 & 0 & 0.0 & - & \\
\hline Playa Larga & 7 & 1 & 8.3 & - & \\
\hline Soplillar & 12 & 2 & 16.6 & - & \\
\hline Los Sábalos & 10 & 2 & 16.6 & 33.3 & \\
\hline Cienfuegos & 27 & 4 & 33.3 & 33.3 & \\
\hline Buey Arriba & 20 & 3 & 25.0 & 25.0 & \\
\hline Gironcito & 15 & 2 & 16.6 & - & $\begin{array}{c}\text { P. florentinoi } \\
16.6\end{array}$ \\
\hline
\end{tabular}

Tabla 5. Heterocigosidad observada (Hobs) por población y por especie.

Localidades: Soroa (SR); Viñales (VI); Sabinal (SA); Cayo Coco (CC); Punta del Este (PE); Cocodrilo (CO); Playa Girón (PG); Playa Larga (PL); Soplillar (SP); Los Sábalos (LS); Cienfuegos (CF); Buey Arriba (BA); Gironcito(Gl).

\begin{tabular}{|c|c|c|c|c|c|c|c|c|c|c|c|c|c|}
\hline & \multicolumn{2}{|c|}{ P. fustiger } & \multicolumn{10}{|c|}{ P. peltocephala } & P. florentinoi \\
\hline Locus & SR & $\mathrm{VI}$ & SA & CC & $\mathrm{PE}$ & $\mathrm{CO}$ & $P G$ & $\mathrm{PL}$ & SP & LS & $\mathrm{CF}$ & BA & GI \\
\hline PGM & 0.250 & 0.111 & 0.000 & 0.200 & 0.800 & 0.500 & 0.000 & 0.000 & 0.250 & 0.200 & 0.053 & 0.250 & 0.000 \\
\hline AAT-B & 0.250 & 0.111 & 0.294 & 0.000 & 0.000 & 0.000 & 0.000 & 0.000 & 0.000 & 0.000 & 0.100 & 0.200 & 0.400 \\
\hline LDH-B & 0.180 & 0.222 & 0.000 & 0.000 & 0.600 & 0.429 & 0.000 & 0.286 & 0.250 & 0.200 & 0.200 & 0.000 & 0.000 \\
\hline $\mathrm{MDH}-\mathrm{A}$ & 0.210 & 0.000 & 0.000 & 0.000 & 0.000 & 0.000 & $0-000$ & 0.000 & 0.000 & 0.000 & 0.150 & 0.050 & 0.067 \\
\hline Hobs & 0.074 & 0.037 & 0.024 & 0.016 & 0.117 & 0.077 & 0.000 & 0.024 & 0.042 & 0.033 & 0.042 & 0.042 & 0.039 \\
\hline Hobs & \multicolumn{2}{|c|}{0.056} & \multicolumn{10}{|c|}{0.042} & 0.039 \\
\hline
\end{tabular}

El grado de diversificación que tienen las subpoblaciones dentro de la metapoblación (Gst) resultó mayor en los Cayos como era de esperar, por el relativo aislamiento geográfico que presentan entre ellas. Las poblaciones de Cayo Sabinal y Cayo Coco se diferenciaron entre sí en 6.8\%.

Las poblaciones de Playa Larga, Playa Girón, Soplillar y Los Sábalos, de la Ciénaga de Zapata, se diferencian entre ellas en 5.7\%. Punta del Este y Cocodrilo, subpoblaciones de la Isla de la Juventud se diferencian en $4.0 \%$. 
Los valores anteriores reflejan, a su vez, la tasa de migración (Nem) que resultó ser mayor en la Isla de la Juventud (5.8), o sea, el flujo genético estimado entre las subpoblaciones de cada metapoblación fue de 5 a 6 individuos por generación, seguido por Ciénaga de Zapata (4.1) y los Cayos (3.4).

El bajo valor de flujo genético que presentó la metapoblación de los Cayos (3.4) está relacionado con el bajo valor de heterocigosidad total, es decir, la subdivisión de la población favorece un mayor número de homocigotos, con la consiguiente disminución de los heterocigotos y una mayor diferenciación de sus poblaciones.

Número promedio de alelos por locus. Los valores del número promedio de alelos por locus (A) considerando las especies muestran para $P$. peltocephala un intervalo entre 1.00 y 1.42 , para $P$. fustiger entre 1.25 y 1.60 y para $P$. florentinoi un valor de 1.20 .

Por poblaciones, Playa Girón tiene el valor más bajo con 1.00. Cayo Sabinal, Cayo Coco y Playa Larga tienen un valor idéntico de 1.10, Soplillar presenta 1.20, mientras Punta del Este, Cocodrilo y Los Sábalos tienen un valor ligeramente mayor (1.25). Cienfuegos presenta un valor de 1.33. El mayor valor de número promedio de alelos por locus para P. peltocephala fue de 1.42 (Buey Arriba).

Por especies, $P$. fustiger mostró el mayor valor de A con 1.40, seguido por $P$. peltocephala, con 1.32 y por $P$. florentinoi con 1.20 .

Distancia genética. El fenograma obtenido a partir de la matriz de distancias genéticas (D) de NEI (1972), calculada sobre la base de las frecuencias génicas (Tabla 6), con un coeficiente de correlación cofenética $(r=0.97)$ elevado, presentó poca distorsión de su configuración espacial y refleja fielmente las relaciones entre los grupos formados. En el fenograma (Fig. 2) se observa que la principal dicotomía separa claramente a $P$. florentinoi del resto de las unidades taxonómicas. La segunda dicotomía principal permite establecer dos agrupamientos claramente definidos. El primero está formado por las dos poblaciones de $P$. fustiger (Soroa y Viñales) y el segundo por poblaciones de $P$. peltocephala, dentro del que hay un subagrupamiento formado por las poblaciones de la Isla de la Juventud (Punta del Este y Cocodrilo) y el otro por las poblaciones de los Cayos, Ciénaga de Zapata, Cienfuegos y Buey Arriba.

$P$. fustiger y $P$. peltocephala difieren con un valor promedio de $D=0.098$ y $P$. florentinoi difiere de ambas en una $\mathrm{D}=0.197$. Las poblaciones de $P$. peltocephala de la Isla de la Juventud se separan del resto en un valor promedio de $D=0.019$. mientras que las de los Cayos, Ciénaga de Zapata, Cienfuegos y Buey Arriba presentan un alto grado de similaridad genética. 
Tabla 6. Matriz de Distancia genética Nei (1972). (Coeficiente de correlación cofenética, $r=0.97)$. Localidades: Soroa (SR); Viñales (VI); Sabinal (SA); Cayo Coco (CC); Punta del Este (PE); Cocodrilo (CO); Playa Girón (PG); Playa Larga

(PL); Soplillar (SP); Los Sábalos (LS); Cienfuegos (CF); Buey Arriba (BA); Gironcito (Gl).

\begin{tabular}{|l|l|l|l|l|l|l|l|l|l|l|l|l|l|}
\hline & SR & VI & SA & CC & PE & CO & PG & PL & SP & LS & CF & BA & GI \\
\hline SR & 0.000 & & & & & & & & & & & & \\
\hline VI & 0.002 & 0.000 & & & & & & & & & & & \\
\hline SA & 0.095 & 0.093 & 0.000 & & & & & & & & & & \\
\hline CC & 0.095 & 0.091 & 0.003 & 0.000 & & & & & & & & & \\
\hline PE & 0.116 & 0.114 & 0.029 & 0.023 & 0.000 & & & & & & & & \\
\hline CO & 0.111 & 0.107 & 0.021 & 0.017 & 0.008 & 0.000 & & & & & & & \\
\hline PG & 0.094 & 0.090 & 0.002 & 0.001 & 0.027 & 0.019 & 0.000 & & & & & & \\
\hline PL & 0.095 & 0.090 & 0.004 & 0.003 & 0.021 & 0.014 & 0.002 & 0.000 & & & & & \\
\hline SP & 0.098 & 0.093 & 0.007 & 0.005 & 0.014 & 0.006 & 0.005 & 0.002 & 0.000 & & & & \\
\hline LS & 0.095 & 0.091 & 0.003 & 0.001 & 0.018 & 0.011 & 0.001 & 0.001 & 0.001 & 0.000 & & & \\
\hline CF & 0.094 & 0.090 & 0.002 & 0.001 & 0.023 & 0.016 & 0.001 & 0.001 & 0.003 & 0.001 & 0.000 & & \\
\hline BA & 0.094 & 0.092 & 0.002 & 0.001 & 0.022 & 0.017 & 0.002 & 0.003 & 0.006 & 0.002 & 0.001 & 0.000 & \\
\hline GI & 0.289 & 0.279 & 0.188 & 0.192 & 0.168 & 0.161 & 0.190 & 0.165 & 0.158 & 0.174 & 0.177 & 0.193 & 0.000 \\
\hline
\end{tabular}

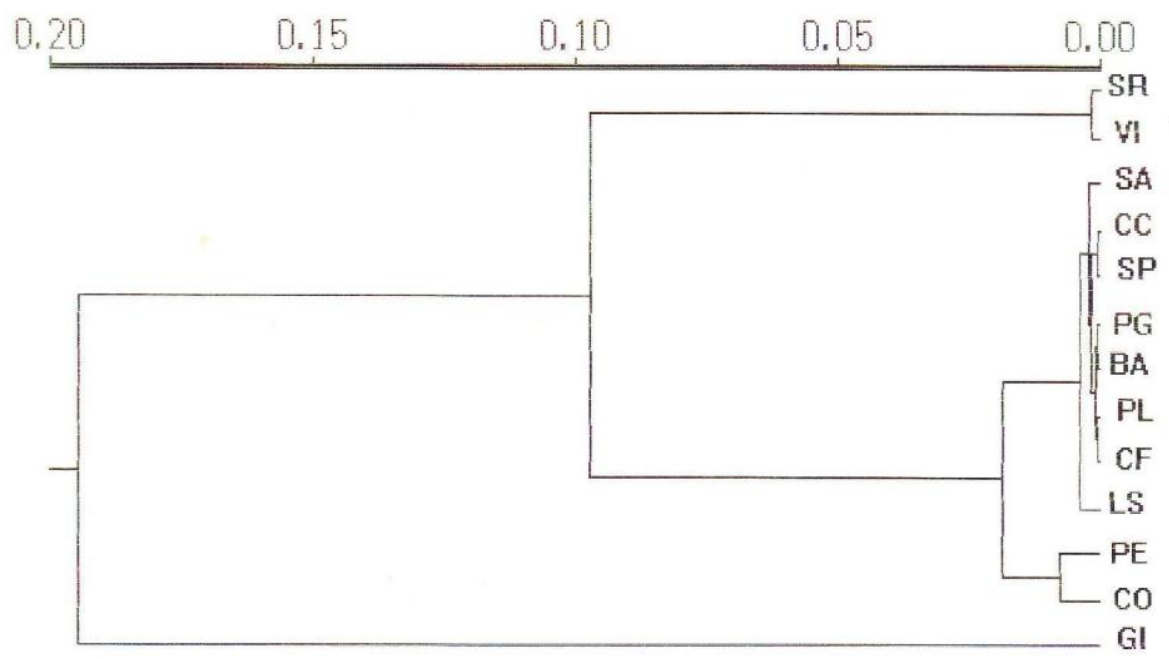

Figura 2. Dendrograma de distancia genética (Nei, 1972). Localidades: Soroa (SR); Viñales (VI); Sabinal (SA); Cayo Coco (CC); Punta del Este (PE); Cocodrilo (CO); Playa Girón (PG); Playa Larga (PL); Soplillar (SP); Los Sábalos (LS); Cienfuegos (CF); Buey Arriba (BA); Gironcito (Gl). 


\section{Discusión}

Comparación de las frecuencias génicas. En $P$. florentinoi no existe el alelo $\underline{\mathrm{C}}$ en el locus AAT-B y en el locus LDH-B el alelo $\underline{b}$ está fijado, lo que la separa de las otras dos especies. La heterogeneidad genética es significativamente alta dentro de las poblaciones de $P$. peltocephala y de $P$. fustiger para los loci PGM, AAT-B y LDH-B. Este fenómeno pudiera ser explicado, bien por una microdiferenciación genética local debido a las particularidades ambientales de cada población, o bien por la acción de la deriva genética a lo largo de la historia demográfica de las poblaciones (SELANDER y KAUFMAN, 1975; MITTON, 1997).

Grado de polimorfismo. En la comparación por áreas geográficas, se destaca el hecho de que el valor más bajo de $P=16.6 \%$ se corresponde con áreas insulares (Cayo Coco, Cayo Sabinal e Isla de la Juventud) lo cual está en concordancia con la literatura, donde se plantea que las poblaciones insulares, dado su pequeño tamaño efectivo, deben tener una menor variabilidad genética (NEVO, 1978; AVISE y HAMRICK, 1996).

$P$. peltocephala y $P$. fustiger presentan un valor promedio de $P=25.0 \%$ y $29.1 \%$, respectivamente, $y$ ambas se acercan al valor promedio para anfibios $\mathrm{P}=26.9 \%$ (NEVO, 1978) y $\mathrm{P}=28.4 \%$ (NEVO et al., 1984).

Heterocigosidad. El nivel de heterocigosidad registrado por NEVO y BEILES (1991) para siete anuros tropicales tiene un promedio de $0.069 \pm 0.055$. Este promedio es algo superior a los encontrados en nuestro trabajo, lo que pudiera estar condicionado por el tamaño de las muestras y número de loci analizados, por la condición de insularidad y de especialización del hábitat, o todo a la vez.

Descomposición de la heterocigosidad por metapoblación en $\boldsymbol{P}$. peltocephala. El flujo genético es causa de la disminución de las diferencias genéticas entre grupos. Es característico de algunas especies de anfibios, el agregarse en el momento de la reproducción (ZUG et al., 2001), fenómeno que contribuye de manera efectiva al flujo genético, ya que los migrantes se reproducen en nuevas áreas o retornan a reproducirse a su lugar de origen.

En resumen, los mayores valores de variabilidad genética se encontraron en las poblaciones de la Isla de la Juventud. Por otro lado, se presentó una menor diferenciación de estas poblaciones, lo cual se traduce en un mayor flujo genético 
debido probablemente a que Punta del Este y Cocodrilo estén separadas por poblaciones intermedias que mantienen la continuidad del flujo genético.

Los Cayos resultan los de menor variabilidad genética y mayor diferenciación entre las subpoblaciones. Este resultado es lógico ya que en los cayos el aislamiento geográfico, dado por barreras marinas, limita la dispersión de los individuos entre las poblaciones consideradas (RODRÍGUEZ-SCHETTINO, 1993). La metapoblación de la Ciénaga de Zapata presenta una situación intermedia, pues tiene heterocigosidad baja y sus poblaciones están poco diferenciadas: La baja heterocigosidad total para esta zona puede ser consecuencia de que entre las localidades (Playa Larga, Playa Girón, Soplillar y Los Sábalos) no existen barreras geográficas que impidan el flujo genético, al igual que sucede en la Isla de la Juventud (Fig. 2), y son zonas más antropizadas que las de los Cayos.

Algunas de las heterocigosidades promedio de las poblaciones y especies estudiadas están cercanas a las registradas por MITTON (1997) en vertebrados, para habitat especialistas $(0.037 ; P$. florentinol) y especies endémicas y relictus (0.045; P. florentinoi y P. peltocephala), lo cual en el último caso evidencia, por la vía molecular, la necesidad de protección de estas especies.

Distancia genética. El fenograma derivado de la variación enzimática y proteínica coincidió plenamente con la clasificación taxonómica establecida sobre la base de caracteres morfológicos (VALDÉS, 1988; SCHWARTZ y HENDERSON, 1991; MORENO y RIVALTA, 2007), acústicos y larvales (DÍAZ y CÁDIZ, 2008) y moleculares (RATO et al. en prensa), ya que las poblaciones de las diferentes especies quedaron en agrupamientos bien delimitados.

El valor promedio de distancia genética de las poblaciones de los Cayos, Ciénaga de Zapata, Cienfuegos y Buey Arriba (de 0.001 a 0.002) está en un intervalo interpoblacional (SELANDER y JOHNSON, 1973). Sin embargo, las poblaciones de la Isla de la Juventud $(D=0,019)$ pudieran ser tratadas como una subespecie de $P$. peltocephala. AYALA (1975) plantea que debe existir un valor de $\mathrm{D}=0.031 \pm$ 0.007 para hacer consideraciones a nivel de subespecie; no obstante, señala que la distancia promedio o similaridad varía grandemente entre los diversos grupos y que esta también depende del estado de divergencia evolutiva. También la distancia genética ha sido muy utilizada para delimitar especies o subespecies (LIZANA et al., 1997) aunque no se deben utilizar esquemáticamente. 


\section{Agradecimientos}

A L. V. Moreno García, Ángel Daniel Álvarez, Arturo Hernández Marrero, Julio Novo, Miguel Osorio, Adela Torres, Mercedes Martínez Reyes, Michel Domínguez, Julio Guillermo Calvear, Jesús Casí, Julio Larramendi y Norberto Jiménez por la participación en los viajes de colecta; a Mercedes Martínez Reyes e lleana Fernández García, por la lectura crítica del manuscrito y sus valiosas recomendaciones.

\section{Referencias}

AVISE, J. C. 1994. Molecular markers, natural history and evolution. Chapman and Hall, New York.

AVISE, J. C.; HAMRICK, J. L. 1996. Conservation Genetics. Chapman and Hall, New York.

AYALA, F. J. 1975. Genetic dfferentiation during the speciation process. Evolutionary Biology 8:1-78.

BREWER, G. J. 1970. An Introduction to Isozyme Techniques. Academia Press, New York.

CLAYTON, J. W.; TRETIAK, D. N. 1972. Amine citrate buffers for $\mathrm{pH}$ control in starch gel electrophoresis. Journal of Fishery Research Board of Canada. 29:11691172.

DÍAZ, L. M.; CÁDIZ, A. 2008. Guía taxonómica de los anfibios de Cuba. Abc Taxa 4, Bélgica.

FROST D. R.; GRANT, T.; FAIVOVICH, J. N.; BAIN, R. H.; HAAS, A.; HADDAD, C. F. B.; DE SÁ, R. O.; CHANNING, A.; WILKINSON, M.; DONNELLAN, S. C.; RAXWORTHY, C. J.; CAMPBELL, J. A.; BLOTTO, V. L.; MOLER, P.; DREWS, R C.; NUSSBAUM, R. A.; LYNCH, J. D.; GREEN, D. M.; WHEELER, W. C. 2006. The amphibian tree of live. Bulletin of the American Museum of Natural History 297:1-370. 
HARRIS H.; HOPKINSON, D. A. 1976. Handbook of enzyme electrophoresis in human genetics. North-Holland, Amsterdam.

HEDGES, S. B. 1996. The origin of West Indian amphibians and reptiles. En Contributions to West Indian Herpetology: A Tribute to Albert Schwartz (R. Powell y R. W. Henderson, eds.). Society for the Study of Amphibians and Reptiles, Ithaca, New York. Contributions to Herpetology vol. 12, pp. 95-128.

HEDRICK, P. W. 2000. Genetics of Populations. Jones and Bartlett Publ. Boston Mass.

LIZANA, M.; MACHORDOM, A.; SÁNCHEZ-HERNAIZ, M. J.; SÁNCHEZ, B. 1997. Genetic and morphological structure of Iberian Bufo bufo populations. En, Herpetology'97, Praga, República Checa. Abstracts, Third World Congress of Herpetology, p.129.

LOWE, A.; HARRIS, S.; ASHTON, P. 2004. Ecological Genetics: Desing, Analysis and Application. Blackwell Publ. Malden.

MITTON, J. B. 1997. Selection in Natural Population. Oxford University Press, New York.

MORENO, L. V.; RIVALTA, V. 2007. Especie nueva de sapo del género Bufo (Anura: Bufonidae) de la Península de Zapata, Cuba. Solenodon 6:60-69.

NEI, M. 1972. Genetic distance between populations. American Naturalist 106:283292.

NEI, M. 1987. Molecular Evolutionary Genetics. Columbia Univ. Press, New York.

NEVO, E. 1978. Genetic variation in natural populations: problems and theory. Theoretical Population Biology 13:121-177.

NEVO, E.; BEILES, A. 1991. Genetic diversity and ecological heterogeneity in amphibian evolution. Copeia, 1991:564-592.

NEVO, E.; BEILES, A.; BEN-SHLOMO, R. 1984. The evolutionary significance of genetic diversity: Ecological demographic and life history correlates. pp.13-213. En Evolutionary dynamics of genetic diversity. (G. S. Mani, ed.). 
POWELL, R.; HENDERSON, R. W; ADLER, K; DUNDEE, H. A. 1996. An annotated cheklist of West Indian amphibians and reptiles. En Contributions to West Indian Herpetology: A Tribute to Albert Schwartz (R. Powell y R. W. Henderson, eds.). Society for the Study of Amphibians and Reptiles, Ithaca, New York. Contributions to Herpetology vol. 12, pp. 51-93 + 8 láms.

PRAMUK, J. B. 2000. Prenasal bones and snout morphology in West Indian Bufonids and the Bufo granulosus species group. Journal of Herpetology 34 (2): 334-340.

PRAMUK, J. B. 2006. Pkylogeny of South American Bufo (Anura: Bufonidae) inferred from combined evidence. Zoological Journal of the Linnean Society 146:407-452.

PRAMUK, J. B., HASS, C. A.; HEDGES, S. B. 2001. Molecular phylogeny and biogeography of West Indian toads (Anura: Bufonidae). Molecular Phylogenetics and Evolution 20 (2):294-301.

PRAMUK, J. B.; ROBERTSON, T; SITES, J. K; NOONAN, B. P. 2007. Around the World in 10 million years: Biogeography of the nearly cosmopolitan true toads (Anura: Bufonidae). Global Ecology and Biogeography 17:72-83.

PREGILL, G. K. 1981. Cranial morphology and evolution of the West Indian toads (Salientia: Bufonidae): resurrection of the genus Peltophryne Fitzinger. Copeia 2:273-285.

RIDGWAY, G. J.; SHERBURNE, S, W.; LEWIS, R. D. 1970. Polymorphism in the esterases of Atlantic herring. Transactions of American Fishery Society 99:147151.

RODRÍGUEZ SCHETTINO, L. 1993. Áreas faunísticas de Cuba según la distribución ecogeográfica actual y el endemismo de los reptiles. Poeyana 436:117.

SCHWARTZ, A.; HENDERSON, R.W. 1991. Amphibians and reptiles of the West Indies: Descriptions, distributions, and natural history. Univ. Florida Press, Gainesville.

SELANDER, R. K; JOHNSON, W. E. 1973. Genetic variation among vertebrate species. Annual Review of Ecololy and Systematics 4:75-91. 
SELANDER, R. K.; KAUFMAN, D. W. 1975. Genetic structure of populations of the brown snail (Helix aspersa). Microgeographic variation. Evolution 29:385-401.

SELANDER, R. K.; SMITH, M. H.; YANG, S. Y.; JOHNSON, W. E.; GENTRY, J. B. 1971. Biochemical polymorphism and systematics in the genus Peromyscus. I. Variation in the old-field Mouse (Peromyscus polionotus). Studies in Genetics VI. University of Texas Publications 7103:49-90.

SHAW, C. R.; PRASAD, R. 1970. Starch gel electrophoresis for enzymes. A compilation of recipes. Biochemical Genetics 4:297-320.

SMITHIES, O. 1955. Zone electrophoresis in starch gels: Group variations in the serum proteins of normal individuals. Biochemical Journal 51:629-641.

SNEATH, P. H. A.; SOKAL, R. R. 1973. Numerical Taxonomy. W. H. Freeman, San Francisco.

SOKAL, R. R.; ROLF, F. J. 1981. Biometry. W. H. Freeman, San Francisco.

VALDÉS, A. 1988. Systematic comments on Peltophryne peltocephala (Anura: Bufonidae) in the Cuban Archipielago. Caribbean Journal of Sciences 24(1-2):3943.

WRIGHT, S. 1965. The interpretation of population structure by F-statistics with special regard to systems of mating. Evolution 19:395-420.

ZUG, G. R.; VITT, L. J.; CALDWELL, J. P. 2001. Herpetology. An Introductory Biology of Amphibians and Reptiles. Segunda Edición. Academic Press, San Diego, California. 